\title{
A Realistic Estimate of Annual Typical Daily Solar Photovoltaic Power in Urban Armidale NSW, Australia
}

\author{
Yasser Maklad ${ }^{1}$ \\ ${ }^{I}$ (University of New England, Armidale NSW 2351 NSW Australia-School of Environmental \& Rural Science - \\ email:ymaklad@myune.edu.au)
}

\begin{abstract}
Solar energy is one of the common sources of renewable and clean energy. It is really difficult to know exactly the solar radiation at any specific moment at a specific location as clouds are moving simultaneously. Clouds decays solar radiation dramatically. It is astonishingly that in different solar radiation observations are recorded in the same location or town or city due, at the same moment of time, due to the clouds' effect. Such effect has a great influence on solar photovoltaic energy can be generated at any place during the daytime. Generally, manufacturers and suppliers of solar photovoltaic energy system considers the global solar radiation known and published for each town or city as metrological data. However, this led to and overestimation of the solar photovoltaic produced energy and represents a misleading to customers as such estimate it doesn't consider cloudy days.

Armidale is a regional city in New South Wales (NSW) in Australia, as well it is the highest town all over Australia. This study aims to provide a justified realistic/creditable/ reliable estimate of annual typical daily solar photovoltaic power. The reason of this justification that this study depends on an accurate estimate of cloudy global solar radiationfor urban Armidale. Such estimate would be a great benefit of solar energy specialists and non-specialists households to obtain an estimate of daily potential solar photovoltaic power would likely be generated in Armidale.
\end{abstract}

Keywords: - Armidale NSW, solar Photovoltaic power, test meteorological year, test reference year, solar radiation, micro-scale energy generation

\section{INTRODUCTION}

Solar energy is a renewable inexhaustible clean source of energy which was used to meet residential needs of hot water, space heating and cooling, industrial needs of process heat, and agricultural needs of irrigation, drying and cooking. In general, solar energy resource is versatile and does make a significant contribution to the global energy requirements and has the premise of being one of the few, future, clean and reliable energy sources available to mankind [1, $2 \& 3]$.

There are two main categories of solar collection options - direct and indirect. The direct delivery options do not normally require storage or fossil fuel support for small-scale applications but they would for large-scale isolated applications. Such back-up or storage could take any of several forms depending on the application, e.g. batteries, electric network or, if the desired output is heat, internal energy stored in a thermal mass to which heat can be transferred for use at a less favorable time [4 \& 5].

There are various solar energy technologies such as Passive thermal systems for comfort conditions, stationary solar collector systems, solar ponds, tracking collectors, photovoltaic generators (terrestrial) and satellite power systems [6].

This study focuses on the generation of photovoltaic energy in urban Armidale NSW, Australia as a sample of regional Australian cities. This scope is selected mainly to provide micro-scale photovoltaic energy generation systems' designers an annual realistic mean daily photovoltaic power.

\section{DATA AND LOCATION}

A previous study generated the typical meteorological year for global solar radiation [7]. This study was based on daily global solar radiations recorded during the period 1980-2012 are utilized to generate the typical solar radiation data. In Australia, meteorological observations are recorded by the Australian Bureau of Meteorology (BOM) weather stations are widely spreader in lots of cities and towns around Australia. In this study, the global solar radiation data recorded by Armidale Airport Weather Automatic Station and published on the BOM's website where it was collected. The missing and invalid measurements account for approximately $0.01 \%$ of the whole database of global solar radiation; those were replaced with the values of preceding or subsequent days by interpolation. During the calculations process, any year found with more than ten days in any month observations not available was excluded. "Table 1" provides geographical information for Armidale town and the periods of the relevant global solar radiation data. Another previous study considered the effect of cloudy 
days on the annual typical meteorological solar Radiation for Armidale NSW, Australia was developed which is more realistic [8].

Figure 1 shows the location of Armidale city in New South Wales in Australia.

Table 1 Geographical and solar radiation database information of Armidale NSW, Australia

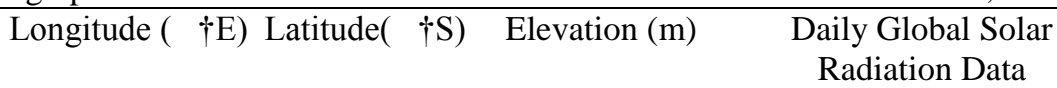

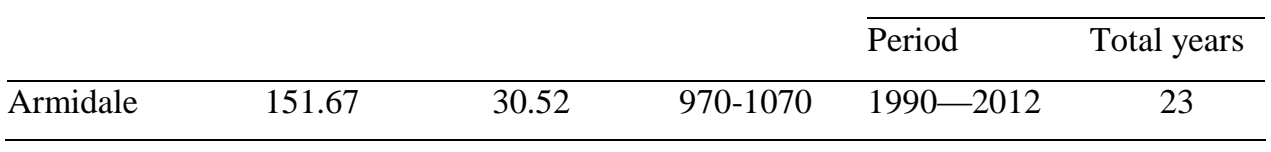

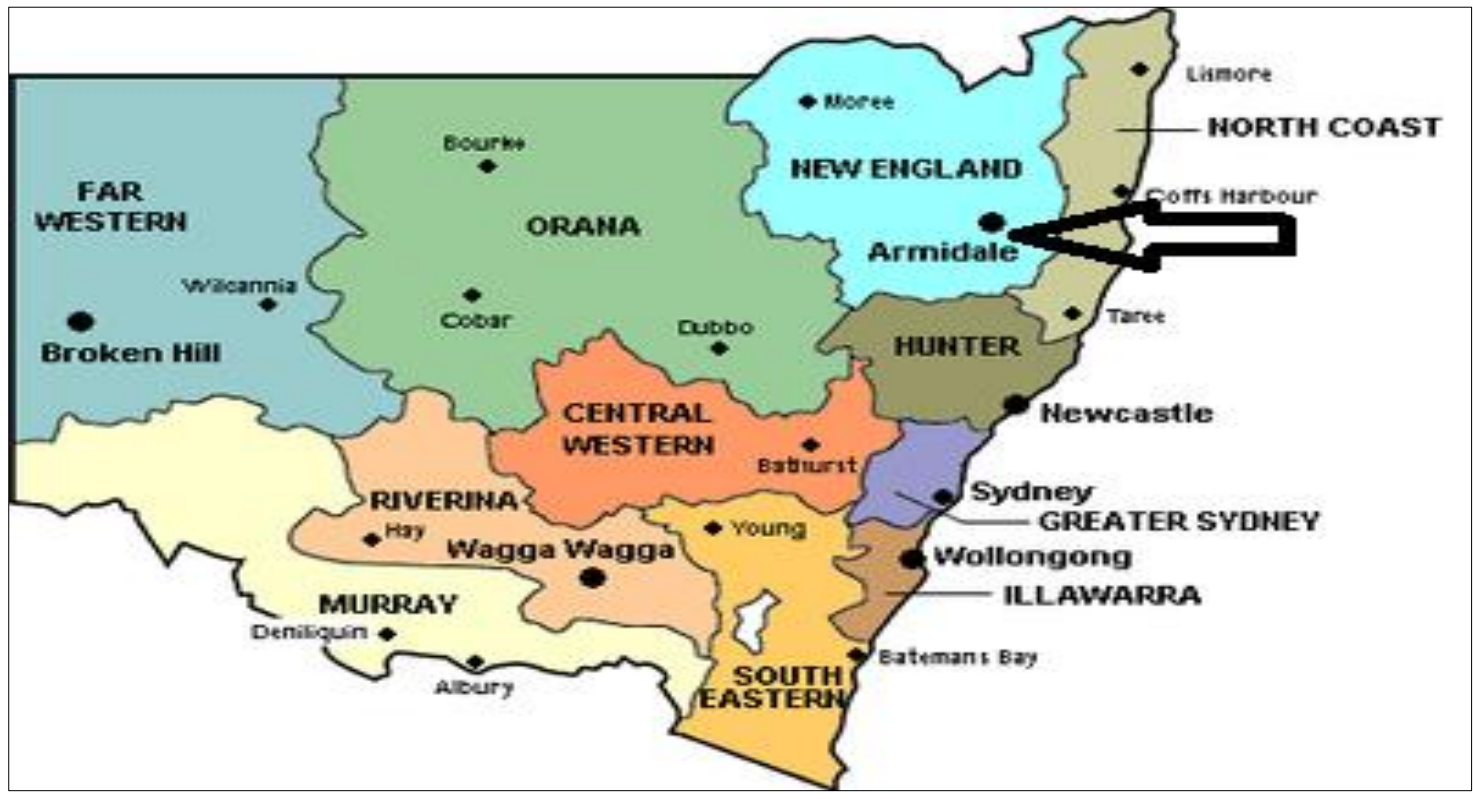

Figure1 Armidale NSW, Australia location

\section{METHODOLOGY}

The power of solar photovoltaic can be estimated by using the following equations [9]:

$$
\mathrm{E}=365 \mathrm{P}_{\mathrm{k}} \mathrm{r}_{\mathrm{p}} \mathrm{H}_{\mathrm{hi}}
$$

Where $\mathrm{E}$ is the yearly potential for electricity generation in kilowatt hours $(\mathrm{kWh}), \mathrm{Pk}$ is the peak power of the equipment installed in kilowatts $(\mathrm{kW}), \mathrm{rp}$ is the system performance ration or dating factor, and Hhi is the monthly or yearly average of daily global radiation in watt-hours (Wh). In the development of the r.sun based PVGIS web calculator, the system performance ratio $\left(r_{p}\right)$ utilized for mono- and polycrystalline silicon panels was 0.75 .

$$
\mathrm{O}_{\mathrm{ut}}=\mathrm{A}_{\mathrm{e}} \mathrm{E}_{\mathrm{e}} \mathrm{G}
$$

Where Eout is the annual electricity production in $\mathrm{kWh}, \mathrm{Ae}$ is the total surface area of solar cells in square meters (m2), Ee is the annual mean power conversion efficiency coefficient for each PV technology, and G is the annual total global irradiation $\left(\mathrm{Wh} / \mathrm{m}^{2}\right)$.

$$
\mathrm{TC}=\mathrm{T}_{\mathrm{amb}}+\left(\mathrm{T}_{0}-20^{\circ} \mathrm{C}\right) \mathrm{E} / 800 \mathrm{Wm}^{-2}
$$

Where $\mathrm{T}_{\mathrm{C}}$ is the photovoltaic panel temperature ${ }^{\circ} \mathrm{C}, \mathrm{T}_{\mathrm{amb}}$ is ambient temperature in degrees Celsius $\left({ }^{\circ} \mathrm{C}\right), \mathrm{T}_{0}$ is the nominal operating cell temperature at ideal conditions ${ }^{\circ} \mathrm{C}$ and $\mathrm{E}$ is the incident radiation in $\mathrm{W} / \mathrm{m}^{2}$ at each time step.

\section{GENERATION OF A TYPICAL ANNUAL MEAN SOLAR PHOTOVOLTAIC POWER}

"Tables2\&3" shows the test meteorological year for global solar radiation and the modified one considered cloudy days in Armidale

Applying the above methodology to "table 3"modified test reference year values it resulted in annual mean daily global solar radiation on a Horizontal Surface in "table 4", peak sunshine sours per day (PSH) in table " 5 " and 
Power in $(\mathrm{kWh})$ per day for a 200w nominal monocrystalline panel of $1.25 \mathrm{~m} 2$ area in "table 6".For simplification, "table 7" shows Power in (kWh) for a 10m2 surface area of 200w nominal monocrystalline panel of $1.25 \mathrm{~m} 2$ area each of total 8 panels with a nominal system capacity $1600 \mathrm{~W}$.

Table 2 Daily global solar radiation values $\left(\mathrm{MJ} / \mathrm{m}^{2}\right.$ day) obtained from Test Reference Year data for Armidale

\begin{tabular}{|c|c|c|c|c|c|c|c|c|c|c|c|c|}
\hline Day & Jan & Feb & Mar & Apr & May & Jun & Jul & Aug & Sep & Oct & Nov & Dec \\
\hline 1 & 29.4 & 18.1 & 19.1 & 18.8 & 13.5 & 10.8 & 10.0 & 14.5 & 16.4 & 20.5 & 22.3 & 24.6 \\
\hline 2 & 27.0 & 22.5 & 21.8 & 18.9 & 12.6 & 10.6 & 10.4 & 14.2 & 16.3 & 19.4 & 22.9 & 25.0 \\
\hline 3 & 27.2 & 23.2 & 21.1 & 17.8 & 13.9 & 9.00 & 11.2 & 13.6 & 16.1 & 19.3 & 23.4 & 26.0 \\
\hline 4 & 26.1 & 26.1 & 22.0 & 19.0 & 13.0 & 10.7 & 10.0 & 13.2 & 17.5 & 20.9 & 23.1 & 25.7 \\
\hline 5 & 23.2 & 25.5 & 19.8 & 16.8 & 12.0 & 10.9 & 12.0 & 13.3 & 15.8 & 21.2 & 21.3 & 25.4 \\
\hline 6 & 23.2 & 23.5 & 21.3 & 19.5 & 14.7 & 11.6 & 11.2 & 14.3 & 18.7 & 19.9 & 22.3 & 23.8 \\
\hline 7 & 26.6 & 21.5 & 19.4 & 18.8 & 14.5 & 9.70 & 10.8 & 13.7 & 17.0 & 21.4 & 24.1 & 26.1 \\
\hline 8 & 25.1 & 23.2 & 20.3 & 16.4 & 12.8 & 10.3 & 10.7 & 14.9 & 17.0 & 21.6 & 22.0 & 26.8 \\
\hline 9 & 24.9 & 23.2 & 19.5 & 16.2 & 13.4 & 10.6 & 9.60 & 14.9 & 15.7 & 22.3 & 23.2 & 24.8 \\
\hline 10 & 26.4 & 24.1 & 21.0 & 17.4 & 12.9 & 10.7 & 11.2 & 15.4 & 18.7 & 21.5 & 24.7 & 22.7 \\
\hline 11 & 26.4 & 24.3 & 21.4 & 16.3 & 12.8 & 10.1 & 11.4 & 15.1 & 18.3 & 22.4 & 24.2 & 24.7 \\
\hline 12 & 26.7 & 21.4 & 20.2 & 16.5 & 12.6 & 11.6 & 10.3 & 15.2 & 18.4 & 22.8 & 25.3 & 26.3 \\
\hline 13 & 26.1 & 22.4 & 21.0 & 16.4 & 12.5 & 9.80 & 9.90 & 15.7 & 20.4 & 23.7 & 24.4 & 28.3 \\
\hline 14 & 25.6 & 20.2 & 21.1 & 14.9 & 12.7 & 10.2 & 10.2 & 15.6 & 19.7 & 21.9 & 26.9 & 25.8 \\
\hline 15 & 25.7 & 19.7 & 21.9 & 16.7 & 12.9 & 10.5 & 11.6 & 15.6 & 19.3 & 23.2 & 23.8 & 25.2 \\
\hline 16 & 26.6 & 20.3 & 21.9 & 15.7 & 12.2 & 10.5 & 12.4 & 14.3 & 19.7 & 23.6 & 22.5 & 27.0 \\
\hline 17 & 25.1 & 23.9 & 19.3 & 15.4 & 11.5 & 11.2 & 12.1 & 14.4 & 19.2 & 22.5 & 21.3 & 28.3 \\
\hline 18 & 25.1 & 22.2 & 20.7 & 16.2 & 11.1 & 10.6 & 10.7 & 14.0 & 19.7 & 23.0 & 21.5 & 26.0 \\
\hline 19 & 22.1 & 21.8 & 20.9 & 15.2 & 12.4 & 9.50 & 12.2 & 14.6 & 20.0 & 21.8 & 25.5 & 27.1 \\
\hline 20 & 28.4 & 22.8 & 20.5 & 15.4 & 12.4 & 9.90 & 12.4 & 13.9 & 19.9 & 23.6 & 24.2 & 26.8 \\
\hline 21 & 27.3 & 23.4 & 19.8 & 16.2 & 11.3 & 10.6 & 12.4 & 15.5 & 19.9 & 22.9 & 21.3 & 28.1 \\
\hline 22 & 25.0 & 21.3 & 17.8 & 15.7 & 11.9 & 10.6 & 12.2 & 14.3 & 21.2 & 24.3 & 24.2 & 26.4 \\
\hline 23 & 23.5 & 23.2 & 18.8 & 16.2 & 12.2 & 10.4 & 12.7 & 12.8 & 21.8 & 24.4 & 23.7 & 27.9 \\
\hline 24 & 25.6 & 23.3 & 20.3 & 14.6 & 12.0 & 11.1 & 11.6 & 16.1 & 20.2 & 21.3 & 25.6 & 24.3 \\
\hline 25 & 20.8 & 19.0 & 19.5 & 14.6 & 12.2 & 11.0 & 10.8 & 16.8 & 21.5 & 22.9 & 25.3 & 26.2 \\
\hline 26 & 25.4 & 21.5 & 17.8 & 14.9 & 11.9 & 10.3 & 9.60 & 16.2 & 20.5 & 20.0 & 26.3 & 26.3 \\
\hline 27 & 25.2 & 21.5 & 19.4 & 13.7 & 12.2 & 9.80 & 11.4 & 15.5 & 21.5 & 21.3 & 26.1 & 19.8 \\
\hline 28 & 27.2 & 25.5 & 17.9 & 13.1 & 11.7 & 10.6 & 11.7 & 15.3 & 21.6 & 26.9 & 26.5 & 26.2 \\
\hline 29 & 26.8 & 18.3 & 18.5 & 15.1 & 11.4 & 11.2 & 13.6 & 14.9 & 22.1 & 25.5 & 25.7 & 28.3 \\
\hline 30 & 27.4 & & 15.8 & 14.9 & 11.5 & 9.70 & 13.3 & 14.0 & 23.7 & 21.3 & 26.0 & 26.1 \\
\hline 31 & 22.8 & & 18.7 & & 11.0 & & 12.8 & 15.9 & & 22.6 & & 28.4 \\
\hline
\end{tabular}

Table 3 A Modified test reference year of global solar radiation considering cloudy days $\left(\mathrm{MJ} / \mathrm{m}^{2}\right.$ day) for Armidale

\begin{tabular}{ccccccccccccccc}
\hline Day & Jan & Feb & Mar & Apr & May & Jun & Jul & Aug & Sep & Oct & Nov & Dec \\
\hline 1 & 29.4 & 1.1 & 5.2 & 18.8 & 0.2 & 10.8 & 6.5 & 14.5 & 16.4 & 2.5 & 22.3 & 2.9 \\
2 & 3.9 & 1.6 & 2.8 & 18.9 & 12.6 & 4.4 & 10.4 & 14.2 & 3 & 2.4 & 22.9 & 9.1 \\
3 & 27.2 & 7.1 & 7.6 & 17.8 & 13.9 & 4.1 & 11.2 & 13.6 & 16.1 & 19.3 & 1.5 & 10.8 \\
4 & 7.5 & 3.1 & 7.2 & 19 & 5.6 & 4.8 & 10 & 2.7 & 17.5 & 4.2 & 1.4 & 25.7 \\
5 & 7.4 & 3.9 & 19.8 & 0.7 & 12 & 10.9 & 12 & 13.3 & 15.8 & 21.2 & 1.8 & 25.4 \\
6 & 23.2 & 7.1 & 21.3 & 7.3 & 14.7 & 2.5 & 6.7 & 14.3 & 18.7 & 0.5 & 10.4 & 6.4 \\
7 & 26.6 & 4.5 & 19.4 & 18.8 & 14.5 & 2.5 & 10.8 & 9.1 & 17 & 1.7 & 7.7 & 0.3 \\
8 & 25.1 & 3.4 & 20.3 & 16.4 & 12.8 & 10.3 & 2.9 & 2.4 & 3 & 21.6 & 8.7 & 26.8 \\
9 & 24.9 & 11.5 & 1.9 & 6.7 & 4.9 & 1.6 & 3.2 & 9.6 & 1.4 & 22.3 & 23.2 & 9.6 \\
10 & 26.4 & 24.1 & 21 & 17.4 & 12.9 & 0.2 & 2.3 & 15.4 & 2.5 & 21.5 & 24.7 & 22.7 \\
11 & 26.4 & 4.9 & 21.4 & 2.9 & 2.6 & 10.1 & 2.7 & 3.6 & 2.8 & 22.4 & 24.2 & 2.3 \\
12 & 26.7 & 5.5 & 20.2 & 16.5 & 4.1 & 11.6 & 2.8 & 15.2 & 5.1 & 22.8 & 7.6 & 2.4
\end{tabular}


A Realistic Estimate of Annual Typical Daily Solar Photovoltaic Power in Urban Armidale NSW,

\begin{tabular}{|c|c|c|c|c|c|c|c|c|c|c|c|c|}
\hline 13 & 26.1 & 2 & 21 & 7.9 & 1.6 & 3.2 & 9.9 & 5.2 & 2.2 & 23.7 & 3.8 & 11.1 \\
\hline 14 & 5.4 & 6.1 & 12.6 & 14.9 & 12.7 & 2.4 & 3.4 & 15.6 & 19.7 & 21.9 & 3.4 & 7.1 \\
\hline 15 & 4.7 & 2.1 & 21.9 & 6.9 & 2.8 & 2.2 & 0.5 & 15.6 & 2 & 4 & 23.8 & 10 \\
\hline 16 & 7.8 & 2.2 & 21.9 & 1.1 & 3.8 & 3.7 & 0.8 & 14.3 & 4.9 & 4.3 & 22.5 & 27 \\
\hline 17 & 3.8 & 7.6 & 6.1 & 6.1 & 1.9 & 3.8 & 12.1 & 3 & 5 & 22.5 & 3 & 5.6 \\
\hline 18 & 1.9 & 22.2 & 7.2 & 16.2 & 1.4 & 1.3 & 3.3 & 1.3 & 4.1 & 2.2 & 6.7 & 3.6 \\
\hline 19 & 4 & 2.1 & 20.9 & 15.2 & 12.4 & 3.5 & 1.5 & 14.6 & 2.9 & 4 & 25.5 & 27.1 \\
\hline 20 & 28.4 & 22.8 & 20.5 & 15.4 & 12.4 & 9.9 & 4.3 & 3.6 & 1.9 & 23.6 & 2.7 & 26.8 \\
\hline 21 & 27.3 & 7 & 1.6 & 16.2 & 3.5 & 10.6 & 4.6 & 15.5 & 19.9 & 22.9 & 2 & 28.1 \\
\hline 22 & 25 & 0.4 & 17.8 & 3.2 & 1.4 & 3.1 & 3.1 & 4.4 & 21.2 & 24.3 & 2.8 & 10.3 \\
\hline 23 & 1.2 & 23.2 & 18.8 & 2.9 & 12.2 & 10.4 & 12.7 & 3 & 21.8 & 4.5 & 3.7 & 27.9 \\
\hline 24 & 25.6 & 23.3 & 20.3 & 14.6 & 4.9 & 11.1 & 5.9 & 5.4 & 1.9 & 1.9 & 25.6 & 24.3 \\
\hline 25 & 6.2 & 3.3 & 8.1 & 14.6 & 2.5 & 11 & 2.8 & 6.6 & 21.5 & 22.9 & 10 & 3.6 \\
\hline 26 & 6.7 & 21.5 & 4.5 & 4.9 & 1.7 & 2.9 & 0.5 & 4.2 & 20.5 & 4.5 & 0.3 & 7.4 \\
\hline 27 & 25.2 & 21.5 & 8 & 4.4 & 2.4 & 9.8 & 0.9 & 2.5 & 21.5 & 21.3 & 26.1 & 4.4 \\
\hline 28 & 27.2 & 25.5 & 4.9 & 2.9 & 11.7 & 3.9 & 2 & 2 & 21.6 & 26.9 & 26.5 & 26.2 \\
\hline 29 & 1.6 & 18.3 & 18.5 & 6.1 & 2.9 & 2.5 & 2.4 & 4 & 22.1 & 25.5 & 25.7 & 28.3 \\
\hline 30 & 2.5 & & 3.5 & 3.3 & 11.5 & 1.7 & 13.3 & 7.6 & 23.7 & 2.3 & 26 & 10.7 \\
\hline 31 & 6.8 & & 18.7 & & 11 & & 12.8 & 1 & & 22.6 & & 28.4 \\
\hline
\end{tabular}

Extracted from [8]

Table 4 Global Solar Radiation on a Horizontal Surface $\left(\mathrm{MJ} / \mathrm{m}^{2}\right.$ day) for Armidale

\begin{tabular}{ccrrrrrrrrrrr}
\hline Day & Jan & \multicolumn{1}{c}{ Feb } & \multicolumn{1}{c}{ Mar } & \multicolumn{1}{c}{ Apr } & \multicolumn{1}{c}{ May } & \multicolumn{1}{c}{ Jun } & \multicolumn{1}{c}{ Jul } & Aug & \multicolumn{1}{c}{ Sep } & Oct & Nov & \multicolumn{1}{c}{ Dec } \\
\hline 1 & 8170 & 310 & 1440 & 5220 & 60 & 3000 & 1810 & 4030 & 4560 & 690 & 6190 & 810 \\
2 & 1080 & 440 & 780 & 5250 & 3500 & 1220 & 2890 & 3940 & 830 & 670 & 6360 & 2530 \\
\hline 3 & 7560 & 1970 & 2110 & 4940 & 3860 & 1140 & 3110 & 3780 & 4470 & 5360 & 420 & 3000 \\
\hline 4 & 2080 & 860 & 2000 & 5280 & 1560 & 1330 & 2780 & 750 & 4860 & 1170 & 390 & 7140 \\
\hline 5 & 2060 & 1080 & 5500 & 190 & 3330 & 3030 & 3330 & 3690 & 4390 & 5890 & 500 & 7060 \\
\hline 6 & 6440 & 1970 & 5920 & 2030 & 4080 & 690 & 1860 & 3970 & 5190 & 140 & 2890 & 1780 \\
\hline 7 & 7390 & 1250 & 5390 & 5220 & 4030 & 690 & 3000 & 2530 & 4720 & 470 & 2140 & 80 \\
\hline 8 & 6970 & 940 & 5640 & 4560 & 3560 & 2860 & 810 & 670 & 830 & 6000 & 2420 & 7440 \\
\hline 9 & 6920 & 3190 & 530 & 1860 & 1360 & 440 & 890 & 2670 & 390 & 6190 & 6440 & 2670 \\
10 & 7330 & 6690 & 5830 & 4830 & 3580 & 60 & 640 & 4280 & 690 & 5970 & 6860 & 6310 \\
\hline 11 & 7330 & 1360 & 5940 & 810 & 720 & 2810 & 750 & 1000 & 780 & 6220 & 6720 & 640 \\
12 & 7420 & 1530 & 5610 & 4580 & 1140 & 3220 & 780 & 4220 & 1420 & 6330 & 2110 & 670 \\
13 & 7250 & 560 & 5830 & 2190 & 440 & 890 & 2750 & 1440 & 610 & 6580 & 1060 & 3080 \\
14 & 1500 & 1690 & 3500 & 4140 & 3530 & 670 & 940 & 4330 & 5470 & 6080 & 940 & 1970 \\
15 & 1310 & 580 & 6080 & 1920 & 780 & 610 & 140 & 4330 & 560 & 1110 & 6610 & 2780 \\
16 & 2170 & 610 & 6080 & 310 & 1060 & 1030 & 220 & 3970 & 1360 & 1190 & 6250 & 7500 \\
\hline 17 & 1060 & 2110 & 1690 & 1690 & 530 & 1060 & 3360 & 830 & 1390 & 6250 & 830 & 1560 \\
18 & 530 & 6170 & 2000 & 4500 & 390 & 360 & 920 & 360 & 1140 & 610 & 1860 & 1000 \\
\hline 19 & 1110 & 580 & 5810 & 4220 & 3440 & 970 & 420 & 4060 & 810 & 1110 & 7080 & 7530 \\
\hline 20 & 7890 & 6330 & 5690 & 4280 & 3440 & 2750 & 1190 & 1000 & 530 & 6560 & 750 & 7440 \\
\hline 21 & 7580 & 1940 & 440 & 4500 & 970 & 2940 & 1280 & 4310 & 5530 & 6360 & 560 & 7810 \\
22 & 6940 & 110 & 4940 & 890 & 390 & 860 & 860 & 1220 & 5890 & 6750 & 780 & 2860 \\
\hline 23 & 330 & 6440 & 5220 & 810 & 3390 & 2890 & 3530 & 830 & 6060 & 1250 & 1030 & 7750 \\
\hline
\end{tabular}


A Realistic Estimate of Annual Typical Daily Solar Photovoltaic Power in Urban Armidale NSW,

\begin{tabular}{rrrrrrrrrrrrr}
24 & 7110 & 6470 & 5640 & 4060 & 1360 & 3080 & 1640 & 1500 & 530 & 530 & 7110 & 6750 \\
25 & 1720 & 920 & 2250 & 4060 & 690 & 3060 & 780 & 1830 & 5970 & 6360 & 2780 & 1000 \\
26 & 1860 & 5970 & 1250 & 1360 & 470 & 810 & 140 & 1170 & 5690 & 1250 & 80 & 2060 \\
27 & 7000 & 5970 & 2220 & 1220 & 670 & 2720 & 250 & 690 & 5970 & 5920 & 7250 & 1220 \\
28 & 7560 & 7080 & 1360 & 810 & 3250 & 1080 & 560 & 560 & 6000 & 7470 & 7360 & 7280 \\
29 & 440 & 5080 & 5140 & 1690 & 810 & 690 & 670 & 1110 & 6140 & 7080 & 7140 & 7860 \\
30 & 690 & & 970 & 920 & 3190 & 470 & 3690 & 2110 & 6580 & 640 & 7220 & 2970 \\
31 & 1890 & & 5190 & & 3060 & & 3560 & 280 & & 6280 & & 7890 \\
\hline
\end{tabular}

Table 5 Peak sunshine sours per day (PSH) for Armidale

\begin{tabular}{|c|c|c|c|c|c|c|c|c|c|c|c|c|}
\hline ay & in & $\mathrm{eb}$ & Mar & $\mathrm{pr}$ & May & Iun & $\mathrm{Jul}$ & $\mathrm{ug}$ & Sep & Oct & Nov & Dec \\
\hline 1 & .17 & 0.31 & 1.44 & 522 & 0.06 & 3 & 1.81 & 4.03 & 4.56 & 0.69 & 6.19 & 0.8 \\
\hline 2 & & & & & & 1.22 & & & & & & 2.53 \\
\hline 3 & & & 11 & 404 & 3.86 & 1.14 & & 270 & 4.47 & & 0.42 & \\
\hline 4 & & & & & & & & & & & & 1.14 \\
\hline 5 & 06 & & 5.5 & 0.19 & & 3.03 & & 69 & & & & 7.06 \\
\hline 6 & 44 & 1.5 & & & & & & & & & 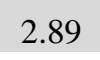 & 1.78 \\
\hline 7 & 39 & 1.25 & 5.39 & 5.22 & 4.03 & 0.69 & 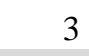 & & & 0.47 & & 0.08 \\
\hline 8 & .97 & 00 & 5.64 & 4.5 & 3.56 & 2.86 & 0.81 & 0.67 & 0.83 & & 2.4 & 7.44 \\
\hline 9 & 22 & 3. & 0.5 & 1.8 & 1. & 0.44 & & & & 6.19 & 6.44 & 2.67 \\
\hline 10 & 33 & 6.6 & 5.83 & 4.83 & 3.5 & 0.06 & 0.64 & 4 & 0.69 & 5.97 & 6. & 6.31 \\
\hline 11 & 33 & 1.3 & 5.94 & & & 2.81 & & & & & & 0.64 \\
\hline 12 & 7.42 & 1.5 & 5.61 & 4.5 & 1. & 3.22 & 0.78 & 4.22 & 1. & 6. & 2. & 0.67 \\
\hline 13 & & & & & & & & & & & & .08 \\
\hline 14 & 15 & 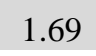 & 3 & 4. & & 0.67 & 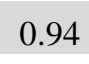 & & & & & 1.97 \\
\hline 15 & & & & & & & & & & & & 2.78 \\
\hline 16 & 2.17 & 0.6 & 6.0 & & & 1.03 & & & & & & $\%$ \\
\hline 17 & 1.06 & 2.1 & 1.69 & 1.69 & 0.53 & 1.06 & 3.36 & 0.83 & 1.39 & 6.25 & 0.83 & 1.56 \\
\hline 18 & & & & & & & & & & & & \\
\hline 19 & 1 & 05 & 5.81 & 4.2 & 3.4 & 0.97 & 0.4 & 406 & 0.8 & 1. & 7.0 & 7.53 \\
\hline 20 & 7.89 & & 5.6 & & & & & & & & & 7.44 \\
\hline 21 & 7.58 & & 0.44 & & 0.97 & 2.94 & 1.28 & 4.31 & & & 05 & 7.8 \\
\hline 22 & 6 & 0 & & & & & & & & & .78 & .86 \\
\hline 23 & & & & & & & & & & & & 7.75 \\
\hline 24 & 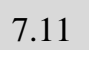 & & & & & & & & & & & 6 \\
\hline 25 & 1.72 & 0.92 & & & 0.69 & 3.06 & & & & 6.36 & 2.78 & \\
\hline 26 & 1.86 & 5.97 & 1.25 & 1.36 & 0.47 & 0.81 & 0.14 & 1.17 & 5.69 & 1.25 & 0.08 & 2.06 \\
\hline 27 & 7 & 5.97 & 2.22 & 1.22 & 0.67 & 2.72 & 0.25 & 0.69 & 5.97 & 5.92 & 7.25 & 1.22 \\
\hline 28 & 7.56 & 7.08 & 1.36 & 0.8 & 3.25 & 1.08 & 0.56 & 0.56 & 6 & 7.47 & 7.36 & 7.2 \\
\hline 29 & 0.44 & 5.08 & 5.14 & & 0.81 & 0.69 & & & 6.14 & 7.08 & 7.14 & 7.8 \\
\hline 30 & 0.69 & & 0.97 & 0.92 & 3.19 & 0.47 & 3.69 & 2.11 & 6.58 & 0.64 & 7.22 & 2.9 \\
\hline 31 & 1.89 & & 5.19 & & 3.06 & & .56 & 0.28 & & 6.28 & & \\
\hline
\end{tabular}


A Realistic Estimate of Annual Typical Daily Solar Photovoltaic Power in Urban Armidale NSW,

Table 6 Power in (kWh) per day for a 200w nominal monocrystalline panel of $1.25 \mathrm{~m}^{2}$ area approximately

\begin{tabular}{|c|c|c|c|c|c|c|c|c|c|c|c|c|}
\hline ay & 1 & b & $\mathrm{ar}$ & $\mathrm{pr}$ & पay & Jun & $\mathrm{ul}$ & & & & Nov & \\
\hline 1 & 24 & 047 & 9 & & & & & 13 & 693 & 05 & 41 & 23 \\
\hline 2 & & & & & & & & & & & & \\
\hline 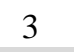 & & & & & & & & & & & & \\
\hline 4 & & & & 803 & & & & & & & & \\
\hline$-\pi$ & & & & & & & & & & & & \\
\hline 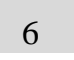 & & & & & & & & & & & & \\
\hline 7 & & & 9 & & & & & & & & & \\
\hline 0 & & & & & & & & & & & & \\
\hline 9 & & & & & & & & & & & & 106 \\
\hline 0 & & & 6 & & & & & & & & & \\
\hline 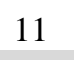 & & & & & & & & & & & & \\
\hline 2 & & 3 & & & & & & & & & & 102 \\
\hline 13 & 2 & .085 & 6 & 3 & 7 & & & & & & & 0 \\
\hline 14 & & & & & & & & & & & & \\
\hline 5 & 0.199 & .088 & 0.924 & 0.292 & 0.119 & 0.093 & & 0.658 & 0.085 & 0.169 & 1.005 & 0.423 \\
\hline & & & & & & & & & & & & \\
\hline 17 & 61 & 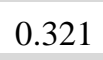 & .257 & 7 & 00 & c & & 6 & & & & 0 \\
\hline & & & & & & & & & & & & \\
\hline 19 & 0160 & 0.088 & 0.883 & & 0.5 & & & & & & & \\
\hline 20 & & & & & & & & & & & & 31 \\
\hline & & & & & & & & & & & & \\
\hline 22 & 1.055 & 017 & .751 & 135 & 0.059 & 1 & 1 & 5 & 895 & 6 & 9 & 35 \\
\hline 23 & & & & & & & & & & & & \\
\hline 24 & 1.081 & 0.983 & 0.857 & 0.617 & 0.207 & 0.468 & 0.249 & 0.228 & 0.081 & & & \\
\hline 25 & & & & & & & & & & & & \\
\hline 26 & 0.283 & .90 & & & 0.071 & & & & & 0.19 & 0.012 & 0.313 \\
\hline 27 & & & & & & & & & & & & \\
\hline 28 & 1.149 & 1.076 & 0.207 & 01 & 0.494 & & 0.085 & 0.085 & 0.912 & 1.135 & 19 & 1.107 \\
\hline 29 & & $0-$ & & & & & & & & & & 95 \\
\hline 30 & & & & & & 71 & & & 1 & 7 & 1.097 & 0. \\
\hline 31 & 0.287 & & 0.789 & & 0.465 & & .54 & .04 & & .93 & & 1.1 \\
\hline
\end{tabular}

Table 7 Power in (kWh) for a $10 \mathrm{~m} 2$ surface area of $200 \mathrm{w}$ nominal monocrystalline panel of $1.25 \mathrm{~m} 2$ area each total panels 8 - Nominal System Capacity $1600 \mathrm{w}$

\begin{tabular}{ccccccccccrrr}
\hline Day & \multicolumn{1}{l}{ Jan } & \multicolumn{1}{l}{ Feb } & \multicolumn{1}{l}{ Mar } & \multicolumn{1}{l}{ Apr } & \multicolumn{1}{l}{ May } & \multicolumn{1}{l}{ Jun } & \multicolumn{1}{l}{ Jul } & \multicolumn{1}{c}{ Aug } & Sep & Oct & \multicolumn{1}{l}{ Nov } & \multicolumn{2}{c}{ Dec } \\
\hline 1 & 9.936 & 0.376 & 1.752 & 6.344 & 0.072 & 3.648 & 2.2 & 4.904 & 5.544 & 0.84 & 7.528 & 0.984 \\
2 & 1.312 & 0.536 & 0.952 & 6.384 & 4.256 & 1.48 & 3.512 & 4.792 & 1.008 & 0.816 & 7.736 & 3.08 \\
3 & 9.192 & 2.392 & 2.568 & 6.008 & 4.696 & 1.384 & 3.784 & 4.6 & 5.432 & 6.52 & 0.512 & 3.648 \\
4 & 2.528 & 1.048 & 2.432 & 6.424 & 1.896 & 1.616 & 3.384 & 0.912 & 5.912 & 1.424 & 0.472 & 8.68 \\
5 & 2.504 & 1.312 & 6.688 & 0.232 & 4.048 & 3.688 & 4.048 & 4.488 & 5.336 & 7.16 & 0.608 & 8.584 \\
6 & 7.832 & 2.392 & 7.2 & 2.472 & 4.96 & 0.84 & 2.264 & 4.824 & 6.312 & 0.168 & 3.512 & 2.168 \\
7 & 8.984 & 1.52 & 6.552 & 6.344 & 4.904 & 0.84 & 3.648 & 3.08 & 5.736 & 0.568 & 2.6 & 0.096 \\
8 & 8.472 & 1.144 & 6.856 & 5.544 & 4.328 & 3.48 & 0.984 & 0.816 & 1.008 & 7.296 & 2.944 & 9.048 \\
9 & 8.416 & 3.88 & 0.648 & 2.264 & 1.656 & 0.536 & 1.08 & 3.248 & 0.472 & 7.528 & 7.832 & 3.248
\end{tabular}


A Realistic Estimate of Annual Typical Daily Solar Photovoltaic Power in Urban Armidale NSW,

\begin{tabular}{rrrrrrrrrrrrr}
10 & 8.912 & 8.136 & 7.088 & 5.872 & 4.352 & 0.072 & 0.776 & 5.208 & 0.84 & 7.256 & 8.344 & 7.672 \\
\hline 11 & 8.912 & 1.656 & 7.224 & 0.984 & 0.872 & 3.416 & 0.912 & 1.216 & 0.952 & 7.56 & 8.168 & 0.776 \\
12 & 9.024 & 1.864 & 6.824 & 5.568 & 1.384 & 3.912 & 0.952 & 5.128 & 1.728 & 7.696 & 2.568 & 0.816 \\
\hline 13 & 8.816 & 0.68 & 7.088 & 2.664 & 0.536 & 1.08 & 3.344 & 1.752 & 0.744 & 8 & 1.288 & 3.744 \\
14 & 1.824 & 2.056 & 4.256 & 5.032 & 4.296 & 0.816 & 1.144 & 5.264 & 6.648 & 7.392 & 1.144 & 2.392 \\
15 & 1.592 & 0.704 & 7.392 & 2.336 & 0.952 & 0.744 & 0.168 & 5.264 & 0.68 & 1.352 & 8.04 & 3.384 \\
\hline 16 & 2.64 & 0.744 & 7.392 & 0.376 & 1.288 & 1.256 & 0.264 & 4.824 & 1.656 & 1.448 & 7.6 & 9.12 \\
17 & 1.288 & 2.568 & 2.056 & 2.056 & 0.648 & 1.288 & 4.088 & 1.008 & 1.688 & 7.6 & 1.008 & 1.896 \\
\hline 18 & 0.648 & 7.504 & 2.432 & 5.472 & 0.472 & 0.44 & 1.12 & 0.44 & 1.384 & 0.744 & 2.264 & 1.216 \\
\hline 19 & 1.352 & 0.704 & 7.064 & 5.128 & 4.184 & 1.176 & 0.512 & 4.936 & 0.984 & 1.352 & 8.608 & 9.16 \\
\hline 20 & 9.592 & 7.696 & 6.92 & 5.208 & 4.184 & 3.344 & 1.448 & 1.216 & 0.648 & 7.976 & 0.912 & 9.048 \\
\hline 21 & 9.216 & 2.36 & 0.536 & 5.472 & 1.176 & 3.576 & 1.56 & 5.24 & 6.728 & 7.736 & 0.68 & 9.496 \\
\hline 22 & 8.44 & 0.136 & 6.008 & 1.08 & 0.472 & 1.048 & 1.048 & 1.48 & 7.16 & 8.208 & 0.952 & 3.48 \\
\hline 23 & 0.4 & 7.832 & 6.344 & 0.984 & 4.12 & 3.512 & 4.296 & 1.008 & 7.368 & 1.52 & 1.256 & 9.424 \\
\hline 24 & 8.648 & 7.864 & 6.856 & 4.936 & 1.656 & 3.744 & 1.992 & 1.824 & 0.648 & 0.648 & 8.648 & 8.208 \\
\hline 25 & 2.088 & 1.12 & 2.736 & 4.936 & 0.84 & 3.72 & 0.952 & 2.224 & 7.256 & 7.736 & 3.384 & 1.216 \\
\hline 26 & 2.264 & 7.256 & 1.52 & 1.656 & 0.568 & 0.984 & 0.168 & 1.424 & 6.92 & 1.52 & 0.096 & 2.504 \\
\hline 27 & 8.512 & 7.256 & 2.696 & 1.48 & 0.816 & 3.304 & 0.304 & 0.84 & 7.256 & 7.2 & 8.816 & 1.48 \\
\hline 28 & 9.192 & 8.608 & 1.656 & 0.984 & 3.952 & 1.312 & 0.68 & 0.68 & 7.296 & 9.08 & 8.952 & 8.856 \\
\hline 29 & 0.536 & 6.176 & 6.248 & 2.056 & 0.984 & 0.84 & 0.816 & 1.352 & 7.464 & 8.608 & 8.68 & 9.56 \\
\hline 30 & 0.84 & & 1.176 & 1.12 & 3.88 & 0.568 & 4.488 & 2.568 & 8 & 0.776 & 8.776 & 3.608 \\
\hline 31 & 2.296 & & 6.312 & & 3.72 & & 4.328 & 0.344 & & 7.64 & & 9.592 \\
\hline Total & 166.21 & 97.52 & 143.47 & 107.42 & 76.19 & 57.66 & 60.26 & 86.90 & 120.81 & 151.37 & 133.93 & 156.18 \\
\hline
\end{tabular}

\section{CONCLUSION AND RECOMMENDATIONS}

Predicting solar photovoltaic power could be generated is a sophisticated task. As clouds directly affects the solar radiation and can decay it during the daytime. This paper considered developing a realistic/reliable/creditable database of annual mead daily typical solar photovoltaic typical daily power database based on previously published generated solar radiation test reference year considering cloudy days in urban Armidale. Such consequent calculations justifies and enhance the reliability of generated solar photovoltaic power. It is a common issue that mostly new photovoltaic systems 'users gets disappointed of their solar systems' underperformance, although the fact that they have overestimated the available solar radiation, actually, there are misled by overestimated meteorological historically recorded solar radiation at their towns or cities. This paper considered Armidale, the highest city in Australia, as a sample of Australian regional cities

Therefore, it is highly urged to base any solar photovoltaic power calculations or modelling on test reference years considering cloudy days. This would reflect the real picture of solar photovoltaic potential and would save any disappointment of not reaching the expected energy generation which is usually misled by any sort of overestimation.

It is worth mentioning that, there is a recently published study provided a realistic estimate of annual typical daily wind power for urban Armidale at heights $(8,9 \& 10)$ meters above ground which suit micro-scale wind turbine installations [10]. Such study, in addition to this one, would provide annual typical daily generated power do both solar and wind energies which is highly recommended to be utilised while studying and designing hybrid solar and wind systems for Armidale NSW. As well, a more specialised study providing an estimate of solar radiation on tilted surfaces of angles $\left(15^{\circ}, 30^{\circ}, 45^{\circ}, 60^{\circ} \& 75^{\circ}\right)$ is recommended to be used by solar photovoltaic specialists and designers when considering solar systems with sun tracking devices [11].

\section{REFERENCES}

[1] Natural Resources Canada, 2001). Photovoltaics for Buildings: Opportunities for Canada. Catalogue Number M39-76/2001E, ISBN 0-662-30106-4.

[2] W. Schmidt, B. Woesten, 2000. Proceedings of the 16th European PV Solar Energy Conference, Glasgow, 1083.

[3] H. Sakata, T. Nakai, T. Baba, M. Taguchi, 2000. Proceedings of IEEE Photovoltaic Specialists 
'Conference, 7-12.

[4] J. Szlufcik, S. Sivoththaman, J. Nijs, R. Mertens, R., Van Overstraeten, 1997. Low-cost Industrial Technologies of Crystalline Silicon Solar Cells. Proceedings of the IEEE 85:711-730.

[5] R. Kawamura, K. Sasatani, T. Onizuka, K. Kameko, 1996. Proceedings of the International PVSEC-9, Miyazaki, Japan, 75-78.

[6] B. Ponomaryov, R. Ward., 1982. Layman's Guide to New and Renewable Sources Energy. International Journal of Solar Energy, 1(5), 379-397.

[7] Y. Maklad, 2014. Generation of an Annual Typical Meteorological Solar Radiation for Armidale NSW Australia. IOSR Journal of Engineering (IOSRJEN): 4(4), 2014, 41-45.

[8] Y. Maklad, 2014. The Effect of Cloudy Days on the Annual Typical Meteorological Solar Radiation for Armidale NSW, Australia. IOSR Journal of Engineering (IOSRJEN): 4(8), 2014, 14-20.

[9] C. Carl., 2014. Calculating Solar Photovoltaic Potential on Residential Rooftops in Kailua Kona, Hawaii. Master of Science Thesis. University of Southern California. USA.

[10] Y. Makald, 2014. A Realistic Estimate of Annual Typical Daily Wind Power of different heights in Urban Armidale NSW, Australia. IOSR Journal of Engineering (IOSRJEN): 4(9).

[11] Y. Maklad, 2014. Generation of an Annual Typical Meteorological Solar Irradiance on Tilted Surfaces for Armidale NSW, Australia. IOSR Journal of Engineering (IOSRJEN): 4(7), 2014, 24-40. 